\title{
Positive selection of skeleton genes during domestication indicates the potential genetic mechanism for loss of flight in ducks
}

\section{Tao Zhu}

China Agricultural University https://orcid.org/0000-0003-4837-0185

\section{Xin Qi}

China Agricultural University

\section{Yu Chen}

Beijing Municipal General Station of Animal Science

\section{Liang Wang}

Beijing Municipal General of Animal Science

\section{Xueze Lv}

Beijing Municipal Science and Technology Commission

\section{Weifang Yang}

Beijing Municipal General Station of Animal Science

Jianwei Zhang

Beijing Municipal General Station of Animal Science

Kaiyang Li

Beijing Municipal General Station of Animal Science

Zhonghua Ning

China Agricultural University

\section{Zhihua Jiang}

Washington State University

Lujiang Qu ( $\square$ quluj@163.com )

China Agricultural University

\section{Research article}

Keywords: loss of flight, duck, FGF gene, EIF2AK3 gene, artificial selection

Posted Date: February 10th, 2021

DOI: https://doi.org/10.21203/rs.3.rs-215271/v1 
License: (c) (i) This work is licensed under a Creative Commons Attribution 4.0 International License. Read Full License 


\section{Abstract \\ Background}

Domestication alters lots of phenotypic, neurologic and physiologic traits between domestic animals and their wild ancestors. Domestic ducks were originated from mallards (Anas platyrhynchos) and some documents also showed that spot-billed ducks (Anas zonorhyncha) could also genetically contribute a small part to the domestication. Compared with the two ancestral species, domestic ducks generally present changes in body size and bone morphology, which is supposed to lead to loss of fight in domestic ducks. In the present study, we performed both genomic and transcriptomic analysis to identify candidate genes in order to elucidate the genetic mechanism underlying the phenotypic variation.

\section{Results}

Our results showed that genes associated with the skeleton systems were positively selected during domestication by Fst analysis between the wild and domestic ducks. We also found that many differentially expressed genes (DEGs) in the breast muscle between the wild and domestic ducks were enriched in the pathway for ossification. Among the genes, FGF14 and EIF2AK3 were also under strong selection by the genomic data, and they were both reported to be associated with limb morphology, bone development and flightlessness in some bird species.

\section{Conclusions}

Our study showed that the skeleton related genes were positively selected in the process of domestication, which could also cause the loss of flight in domestic ducks.

\section{Background}

Domestication is the process that the wild is selected to be the domestic artificially and it changes many traits to fit the requirements of human being. Domestic animals are generally distinguished from their wild ancestors by many aspects, and it is well documented that the genetic architectures are altered dramatically underlying the variation of phenotypic traits (such as morphology in chickens [1, 2], pigs [35] and pigeons [6, 7], feather color [8, 9]), neurology ( such as tameness in companion animals [10-12]) and physiology (such as starch digestibility in dogs [13], reproduction in chicken $[14,15]$ ) between the domestic animals and their wild ancestors.

Loss of flight in some domestic birds (ducks, geese, etc.) is also a common phenotype, which is supposed to be associated with the variation of feather, muscle and skeleton, etc. When wild animals are domesticated for meat or other production traits, mobility is firstly restricted. Pig, cattle, chicken move slower than their wild relatives. Artificial selection also shapes the morphology of animals quickly. 
Modern domestication experiments show that it only took 10 to 15 generations to changes the animal appearance significantly [16]. For example, the body mass of the broiler has been increased by $300 \%$ within 50 years [17]. With the exception of pigeon, all of domesticated birds (chicken, duck, goose, and turkey) are flightless. The morphological changes during domestication are mainly attribute to the loss of flight. Loss of flight can also be caused by similar morphological and physiological changes in birds, and previous studies showed that the body size of flightiness birds is bigger than their flight relatives [18]. Besides, muscle weight, wings and skeletal system are also changed. For instance, a flightless steam duck (T. patachonicus) was founded that wing loading, humerus length, radius length, and ulna length was significantly changed compare to their flight relatives [18], and the similar trend also occurs in flightless rails [19].

The flight ancestor and their flightless offspring provide us a good model to understand genetic mechanisms underlying flightlessness during domestication. Domestic ducks are predominantly originated from mallards (Anas platyrhynchos), and small part from spot-billed ducks [20,21], and lose the capability to flight. Meanwhile, the meat and egg productivities have being increased dramatically in ducks $[8,22]$. Compared to the wild ducks, domesticated ducks evolved variety of feather colors [23, 24], bigger body size and much more egg number [25]. This intense selection changed morphological characteristics and weakened the migration capability of the domesticated ducks.

In the present study, two flight wild duck species and eight flightless domestic duck breeds were selected as model animals to study the potential genetic mechanism which lead to loss of flight in domesticated ducks by genomic and transcriptomic data. And we found that some genes associated with bone development were positively selected and varied by both genomic and transcriptomic levels between the wild and the domestic, which could be an explanation for loss of flight in the ducks.

\section{Result}

\section{Population structure}

In our study, we obtained 38,097,848 high quantity SNPs, and the average depth of SNPs was 564.4. To study the population structure, we calculated top 20 components of our experiment ducks, top 3 components explaining $37.68 \%$ of total variances (Figure $1 \mathrm{a}$ ). The results showed that the wild and the domesticated were classified by a line that component 1 equals 0 , while the component 1 of wild ducks was smaller than 0 (Figure 1a), and all individuals were clustered into 3 groups. PK, CV and ML were clustered into same group. The rest groups were clustered together. In order to understand the phylogenetic relationships of the ducks, the CDS SNPs were used to construct maximum likelihood evolutionary tree, and the phylogenetic tree showed that SB and MD were clustered into one branch, PK, $\mathrm{CV}$, and $\mathrm{ML}$ was clustered into one branch and the rest ducks were grouped into together (Figures 1b).

\section{Skeleton system development genes were positively selected during domestication}


We used Fst algorithm to identity the candidate genes diverged between the wild ducks and the domesticated ducks and a slide window method of Fst was used to detect the related regions ( 0 to 1,0 means no divergence). In order to avoid the bias that caused by Z Chromosomes, we only analyzed autosomes in our study. Finally, we obtained 39,251 autosome windows, and the mean Fst between the wild and domesticated ducks was 0.061 . Two peaks on chromosome 16 and chromosome 8 exceeded 0.2. The maximum Fst value was 0.22 at chromosome 16:300001-350001, which overlapped with TMEM132B gene, and second peak was at chromosome 8:19750001-19800001, overlapping with LOC101797367, also known as CLAC2 (Figure 2). Then, we extracted genes overlapping with top 1\% window or adjacent windows. We identified 541 candidate genes and 260 genes mapped to human genome. Metascape was used to gene function annotation [26]. The results showed that many GO items were related to metabolism and cellular energy conversion (Figure 3a). Interestingly, one GO item GO:0001501 showed significantly correlated to skeleton system development, and 14 genes were contained in this item (Figure 3b).

We also calculated Fst and pi values for each SNP, and the SNPs with pi less than 0.1 and Fst higher than 0.8 were considered as candidates. Totally, 22 SNPs were identified as candidate variants, and the 11 annotated genes were found (table 1). FGF14 gene containing 2 candidate SNPs were found and FGF14 was found to be a bone development gene and lead to flightlessness in many other birds [27-30].

\section{DEGs predominantly enriched in bone developmental processes by RNA-seq data}

Gene function can be present by RNA transcription level in specific time. In our study, breast muscle, liver and brain from the wild and domesticated male ducks were collected for transcriptomic analysis. We compared the expression levels between the wild ducks (mallard) and the domesticated ducks. The reads number for each gene was standardized by FPKM algorithm [31], and differentially expressed genes (DEGs) were identified by fold change and $p$ value. After filtration, we identified 128 genes in liver, 28 genes in brain and 200 genes in breast muscle to be DEGs. The number of DEGs in breast muscle was significantly higher than brain and liver. It indicated that artificial selection of domesticated duck changed muscular structure more significantly. Furtherly, gene ontology (GO) analysis was used for the DEGs of breast muscle, and many of them were categorized on ossification and bone development GO items (Figure 3b). Fibroblast growth factor (FGF) family in the items was reported to be associated with limb development and flight capacity in many species. Three other genes, TMEM132B, FGF14 and FGF6, were expressed in breast muscle as twice high as those in the domestic duck $(p<0.05)$ (Figure 4), but the expression level of CLCA2 gene which was identified as a diverged gene by our Fst test, did not show significant different expression levels in all 3 tissues between the wild and the domestic ducks.

\section{Discussion}

Domestication including captivity and artificial selection has brought great morphological changes in ducks. Meanwhile, breeding also introduced large number of variants into domesticated duck genome. In our study, the wild and domesticated ducks could be distinguished by first principal component (Fig. 1a), 
and it showed that long-term artificial selection brought tremendous variation of duck genomes. In previous study, the differences mainly reflected in agronomic traits, such as egg production, growth rate and fat deposition [8, 22]. The domestic ducks were clustered into two groups by second principal component, corresponding to its biological origin place. Our results suggested that genetic distances in Chinese indigenous duck are related to geographical distances, and indigenous ducks in southern China may originate from a common ancestor.

In our study, the Fst algorithm was employed to identify the diverged genomic regions or genes between the wild and domesticated ducks. In total, 542 genes were identified and $1 \mathrm{GO}$ item contains 14 genes was found related to bone development. Furthermore, the differentially expressed genes (DEGs) in breast muscle also showed that changes of bone development related genes played a key role in the differences between the wild and domestic ducks. Many studies suggested that loss of flight in birds was always accompanied with limb modification and skeletal changes $[18,19,32]$.

Beyond that, we noticed EIF2AK3 gene on chromosome 4 also under selection, and we found EIF2AK3 gene is associated with body size, abnormal skeleton morphology and abnormal hind limb morphology in mouse [33-35]. Morphological changes could contribute to loss of flight in birds. Compared to their flightless relatives, flight birds always have a lighter mass, higher wing area and longer bone length [18, $32,36]$. This change is consistent with the breeding goal of domesticated ducks as body weight is a major breeding goal in ducks. For example, as one of most famous meat-type ducks in the world, the Peking ducks was bred to growth rate and fat deposition, and its body weight can up to $3.1 \mathrm{~kg}$ at 35 day old while the adult mallard is only 1.1 to $1.2 \mathrm{~kg}$ [37-40]. Breeding greatly increased the body weight of ducks, and long-term captivity weakened the importance of wings, which is accompany with the physiologic change. The change may include in flight muscle atrophy, bone strength, relative size of wings (limbs). Therefore, EIF2AK3 gene could be involved in the body weight and limb development and partially lead to flightlessness in ducks.

Considering that slide window algorithm might produce both false positive and false negative results, we also calculated Fst and pi values for each SNP. By this method, 22 SNPs and 11 genes were identified to be diverged. It is noteworthy that one SNP is in a TMTM family gene and 2 SNPs are in FGF14 gene. FGF14 is fibroblast growth factor 14, and the protein encoded by FGF14 is a member of the fibroblast growth factor (FGF) family. FGF family gene has been widely reported associated with flight in animals. Weatherbee et al. found FGF8 gene displayed unique expression in forelimb and hindlimb at embryonic stage, BMP and FGF signaling had a role in the inhibition of interdigital apoptosis [41]. Tokita et al. found FGF10 signaling was involved in wing membrane development and patterning of the wing muscles [42]. Interestingly, previous studies found that FGF10 emanated from the prospective limb mesoderm in birds to serve as an endogenous initiator for limb bud formation, which is associated with the loss of flight in emu (Dromaius novaehollandiae) [43, 44]. In drosophila, components of FGF signaling are expressed in myoblasts, a series of experiments show that FGF is a key factor of the development of flight muscle in drosophila $[45,46]$. Therefore, FGF14 very likely plays an important role in loss of flight in domesticated ducks. 
We also detected the expression level for the diverged genes in liver, breast, and brain. We found that the expression levels of FGF14, FGF6 and TMEM132B in wild ducks were all higher than those in domesticated ducks. This unusual expression difference furtherly supports our hypothesis. We also checked the RNA-seq data for expression levels of the genes including DYRK1A, IFT122, CUX1 and ACOT7, which have previously been identified to be associated with flight in other birds [18, 32, 47, 48]. We did not find expression differences of the genes between the wild and domesticated ducks in our samples (data not shown here). One of explanations is that the genetic mechanisms of loss of flight is different in different species, and it also could be attribute to gene expression spatio-temporal, and many morphology related genes only expressed at embryonic stage.

In our study, by comparing the two ancestral duck species to the domesticated ducks by both genomic and transcriptomic data, we found morphologic genes, especially bone developmental genes, were both positively selected and expressively enriched during domestication, which on one hand satisfied human requirements for food and husbandry, one the other hand led to loss of flight in domestication ducks.

\section{Method}

\section{sampling, genomic and transcriptomic sequencing}

In total, 8 domesticated duck populations and 2 ancestral wild duck populations, mallard (Anas platyrhynchos) and spot-billed ducks (Anas zonorhyncha) were used in our study, and the domesticated duck populations involved Peking duck $(n=8, P K)$, Cherry Valley duck $(n=8, M L)$, Maple Leaf duck $(n=8$, $M L)$, Jinding duck $(n=8, J D)$, Shaoxing duck $(n=8, S X)$, Mei duck $(n=10$, Mei), Shan Ma duck $(n=8, S M)$, Gaoyou duck $(n=8, G Y)$, and the wild ducks included Mallard $(n=21, M D)$ and Spot-billed duck $(n=8, S B)$. Mei, a Chinese indigenous duck, was got from Anhui province, China, and SB was collected from Ningxia province, China. Sample collection was performed by using strict protocols approved by the Animal Welfare Committee of China Agricultural University. The rest of ducks are all from our previous study [8]. Our genomic DNA samples of newly collected samples were extracted using the standard phenol/chloroform extraction method and two paired-end libraries (150bp) were constructed in Illumina Hiseq 2500 sequencing platform. In addition, the tissues of liver, brain and breast muscle from 14 adult male ducks (MD, $n=7 ; P K, n=1 ; C V, n=1 ; M L, n=1 ; J D, n=1 ; S M, n=1 ; S X, n=1 ; G Y, n=1$ ) were chosen for RNA-seq. The detail samples information and sequencing methods can be found in our previous study [8].

\section{Pretreatment of genomic data}

The raw genome data were firstly processed by fastp (v0.20.0) to filter adapter contamination and low quantity reads [49]. Then the clean data were aligned to duck genome (https://www.ncbi.nlm.nih.gov/, accession: GCF_003850225.1) by BWA-MEM (v0.7.15) [50]. Picard (http://broadinstitute.github.io/picard/) was used to sort the aligned data and to remove duplicate reads. Genome Analysis Toolkit v3.6 (GATK, https://gatk.broadinstitute.org/) was used to variant calling and variant filter. SNPs were filtered by following rules: a) QUAL >30.0; b) QD >5.0; c) FS <60.0; d) MQ >40.0; e) 
MQRankSum >-12.5; and f) ReadPosRankSum >-8.0. Additionally, if there were more than 3 SNPs clustered in a 10-bpwindow, all 3 SNPs were considered as false positives and removed [51]. Finally, the highly credible SNPs with VCF format were kept for next analysis [51].

\section{Population structure and phylogenetic trees}

After the SNP file was converted to plink format by VCFtools (v0.1.13)[52], we excluded low quality SNPs with missing rate higher than 0.1 and p-value for Hardy-Weinberg Equilibrium test less than 0.01 [53]. Principal component analysis (PCA) was conducted by plink [54], and top 20 components were used for the population relationship analysis. SNPs that overlapping with CDS region were extracted to construct phylogenetic tree. The SNP file was converted to a phylip file by vcf2phylip (v2.0) [55], and the maximum likelihood phylogenetic tree was constructed by RAxML-NG (v1.0.0) [56]. iTOI was used to visualize the phylogenetic tree [57].

\section{Selective-sweep analysis}

Flight is the most obvious difference between wild and domesticated ducks. In this study, the wild ducks (MD and SB) were put into a group and the rest were clustered into the other group. To minimal the false negative, we used two strategies to identity the candidate diverged genes between them. Firstly, fixation index (Fst) was calculated with $50 \mathrm{~kb}$ windows and slide with $25 \mathrm{~kb}$ step, genome regions overlapping with upstream and downstream $50 \mathrm{~kb}$ of top $1 \%$ Fst windows was considered as candidate region, and gene that overlapping candidate region was consider as candidate selected gene. Then, we estimated pi and Fst values for each SNP, and genes that containing both top $0.01 \%$ Fst and pi value were considered as candidate genes. Fst and pi values were calculated by VCFtools (v0.1.13) [52].

\section{RNA-seq data analysis}

The raw transcriptome data were firstly processed by fastp (v0.20.0)[49]. Then the clean data were aligned to duck genome (https://www.ncbi.nlm.nih.gov/, accession: GCF_003850225.1) by HISAT2 (v 2.1.0) [58]. The aligned file was sorted by samtools ( $v 1.9)$ [50]. Reads number for each gene was counted by featureCounts (1.6.4)[59]. The reads count files were merged by tissue, and the standardization of expression level was calculated by DESeq2 [60]. Genes with absolute value of expression level fold change more than 1.5 and adjusted $p$ value less than 0.05 were considered as differentially expressed genes (DEGs). Metascape was used for gene enrichment analysis [26].

\section{Abbreviations}

CDS: Coding sequences; FST: F-statistics (Fixation indices); DEGs: Differentially expressed genes

\section{Declarations}

\section{Acknowledgements}


We gratefully acknowledge our colleagues in the Poultry Team at the National Engineering Laboratory for Animal Breeding of China Agricultural University, for their assistance on sample collection and data analysis.

\section{Authors' contributions}

LJQ and ZHN conceived the study and participated in its design and coordination. TZ and XQ analyzed the data, YC, LW, XZL, WFY and JWZ contributed resources and funding. LJQ and TZ led the manuscript writing. All authors read and approved the final manuscript.

\section{Funding}

This work was supported by the Beijing Innovation Team of the Modern Agro-industry Technology Research System (BAIC04-2020). The funding bodies played no role in the design of the study and collection, analysis, and interpretation of data and in writing the manuscript.

\section{Availability of data and material}

The data supporting the conclusions of this article are available at NCBI (https://www.ncbi.nlm.nih.gov/, PRJNA686828).

\section{Ethics approval and consent to participate}

Sample collection was performed by using strict protocols approved by the Animal Welfare Committee of China Agricultural University (permit XK622). The experimental procedures with ducks were operated according to the Guidelines for Experimental Animals established by the Ministry of Science and Technology (Beijing, China).

\section{Consent for publication}

Not applicable.

\section{Competing interests}

The authors declare that they have no competing interests.

\section{References}

1. Stange M, Núñez-León $D$, Sánchez-Villagra $M$, Jensen $P$, Wilson L: Morphological variation under domestication: How variable are chickens? ROY SOC OPEN SCI 2018, 5:180993.

2. Duggan BM, Hocking PM, Schwarz T, Clements DN: Differences in hindlimb morphology of ducks and chickens: effects of domestication and selection. GENET SEL EVOL 2015, 47(1):88.

3. Geiger M, Sánchez-Villagra MR: Similar rates of morphological evolution in domesticated and wild pigs and dogs. FRONT ZOOL 2018, 15(1):23. 
4. Neaux D, Sansalone G, Lecompte F, Noûs C, Haruda A, Schafberg R, Cucchi T: Examining the effect of feralization on craniomandibular morphology in pigs, Sus scrofa (Artiodactyla: Suidae). BIOL J LINN SOC 2020, 131(4):870-879.

5. Frantz LAF, Schraiber JG, Madsen O, Megens H, Cagan A, Bosse M, Paudel Y, Crooijmans RPMA, Larson G, Groenen MAM: Evidence of long-term gene flow and selection during domestication from analyses of Eurasian wild and domestic pig genomes. NAT GENET 2015, 47(10):1141-1148.

6. Stringham SA, Mulroy EE, Xing J, Record D, Guernsey MW, Aldenhoven JT, Osborne EJ, Shapiro MD: Divergence, Convergence, and the Ancestry of Feral Populations in the Domestic Rock Pigeon. CURR BIOL 2012, 22(4):302-308.

7. Bartels $\mathrm{T}$ : Variations in the morphology, distribution, and arrangement of feathers in domesticated birds. Journal of Experimental Zoology Part B: Molecular and Developmental Evolution 2003, 298B(1):91-108.

8. Zhang Z, Jia Y, Almeida P, Mank JE, van Tuinen M, Wang Q, Jiang Z, Chen Y, Zhan K, Hou S et al: Whole-genome resequencing reveals signatures of selection and timing of duck domestication. GIGASCIENCE 2018, 7(4).

9. Zhou Z, Li M, Cheng H, Fan W, Yuan Z, Gao Q, Xu Y, Guo Z, Zhang Y, Hu J et al: An intercross population study reveals genes associated with body size and plumage color in ducks. NAT COMMUN 2018, 9(1):2648.

10. Sato DX, Rafati N, Ring H, Younis S, Feng C, Blanco-Aguiar JA, Rubin C, Villafuerte R, Hallböök F, Carneiro $M$ et al: Brain Transcriptomics of Wild and Domestic Rabbits Suggests That Changes in Dopamine Signaling and Ciliary Function Contributed to Evolution of Tameness. GENOME BIOL EVOL 2020, 12(10):1918-1928.

11. Huang S, Slomianka L, Farmer AJ, Kharlamova AV, Gulevich RG, Herbeck YE, Trut LN, Wolfer DP, Amrein I: Selection for tameness, a key behavioral trait of domestication, increases adult hippocampal neurogenesis in foxes. HIPPOCAMPUS 2015, 25(8):963-975.

12. Brusini I, Carneiro M, Wang C, Rubin C, Ring H, Afonso S, Blanco-Aguiar JA, Ferrand N, Rafati N, Villafuerte $\mathrm{R}$ et al: Changes in brain architecture are consistent with altered fear processing in domestic rabbits. Proceedings of the National Academy of Sciences 2018, 115(28):7380.

13. Axelsson E, Ratnakumar A, Arendt M, Maqbool K, Webster MT, Perloski M, Liberg O, Arnemo JM, Hedhammar $\AA$, Lindblad-Toh $\mathrm{K}$ : The genomic signature of dog domestication reveals adaptation to a starch-rich diet. NATURE 2013, 495(7441):360-364.

14. Ericsson M, Fallahsharoudi A, Bergquist J, Kushnir MM, Jensen P: Domestication effects on behavioural and hormonal responses to acute stress in chickens. PHYSIOL BEHAV 2014, 133:161169.

15. Johnsson M, Gustafson I, Rubin C, Sahlqvist A, Jonsson KB, Kerje S, Ekwall O, Kämpe O, Andersson L, Jensen P et al: A Sexual Ornament in Chickens Is Affected by Pleiotropic Alleles at HAO1 and BMP2, Selected during Domestication. PLOS GENET 2012, 8(8):e1002914. 
16. Trut L: Early Canid Domestication: The Farm-Fox Experiment. American Scientist-AMER SC/1999, 87.

17. Knowles TG, Kestin SC, Haslam SM, Brown SN, Green LE, Butterworth A, Pope SJ, Pfeiffer D, Nicol CJ: Leg Disorders in Broiler Chickens: Prevalence, Risk Factors and Prevention. PLOS ONE 2008, 3(2):e1545.

18. Campagna L, McCracken KG, Lovette IJ: Gradual evolution towards flightlessness in steamer ducks*. EVOLUTION 2019, 73(9):1916-1926.

19. Gaspar J, Gibb GC, Trewick SA: Convergent morphological responses to loss of flight in rails (Aves: Rallidae). ECOL EVOL 2020, 10(13):6186-6207.

20. Guo X, Wang ZC, Wang S, Li HF, Suwannapoom C, Wang JX, Zhang C, Shao Y, Wang MS, Jiang RS: Genetic signature of hybridization between Chinese spot-billed ducks and domesticated ducks. ANIM GENET 2020, 51(6):866-875.

21. Li H, Zhu W, Song W, Shu J, Han W, Chen K: Origin and genetic diversity of Chinese domestic ducks. MOL PHYLOGENET EVOL 2010, 57(2):634-640.

22. Gu H, Zhu T, Li X, Chen Y, Wang L, Lv X, Yang W, Jia Y, Jiang Z, Qu L: A joint analysis strategy reveals genetic changes associated with artificial selection between egg-type and meat-type ducks. ANIM GENET 2020, 51(6):890-898.

23. Lee D, Lee J, Heo K, Kwon K, Moon Y, Lim D, Lee K, Kim J: Population analysis of the Korean native duck using whole-genome sequencing data. BMC GENOMICS 2020, 21(1):554.

24. Yu W, Wang C, Xin Q, Li S, Feng Y, Peng X, Gong Y: Non-synonymous SNPs in MC1R gene are associated with the extended black variant in domestic ducks (Anas platyrhynchos). ANIM GENET 2013, 44(2):214-216.

25. Al-Obaidi F, Al-Shadeedi S: Comparison study of egg morphology, component and chemical composition of Mallard duck and domestic Peking duck. Journal of Genetic and Environmental Resources Conservation 2016, 4:5-9.

26. Zhou Y, Zhou B, Pache L, Chang M, Khodabakhshi AH, Tanaseichuk O, Benner C, Chanda SK: Metascape provides a biologist-oriented resource for the analysis of systems-level datasets. NAT COMMUN 2019, 10(1):1523.

27. Tim CR, Bossini PS, Kido HW, Malavazi I, von Zeska Kress MR, Carazzolle MF, Parizotto NA, Rennó AC: Effects of low level laser therapy on inflammatory and angiogenic gene expression during the process of bone healing: A microarray analysis. Journal of Photochemistry and Photobiology B: Biology 2016, 154:8-15.

28. Knowles HJ: Hypoxia-Induced Fibroblast Growth Factor 11 Stimulates Osteoclast-Mediated Resorption of Bone. CALCIFIED TISSUE INT 2017, 100(4):382-391.

29. Overman JR, Helder MN, Ten Bruggenkate CM, Schulten EAJM, Klein-Nulend J, Bakker AD: Growth factor gene expression profiles of bone morphogenetic protein-2-treated human adipose stem cells seeded on calcium phosphate scaffolds in vitro. BIOCHIMIE 2013, 95(12):2304-2313. 
30. Boles RG, Dhamcharee V: Fibroblast Growth Factor Receptor (FGFR) and Bone: Implications for Human Growth. In: Handbook of Growth and Growth Monitoring in Health and Disease. Edited by Preedy VR. New York, NY: Springer New York; 2012: 2871-2888.

31. Mortazavi A, Williams BA, McCue K, Schaeffer L, Wold B: Mapping and quantifying mammalian transcriptomes by RNA-Seq. NAT METHODS 2008, 5(7):621-628.

32. Burga A, Wang W, Ben-David E, Wolf PC, Ramey AM, Verdugo C, Lyons K, Parker PG, Kruglyak L: A genetic signature of the evolution of loss of flight in the Galapagos cormorant. SCIENCE 2017, 356(6341):I3345.

33. Bult CJ, Blake JA, Smith CL, Kadin JA, Richardson JE, The MGDG: Mouse Genome Database (MGD) 2019. NUCLEIC ACIDS RES 2019, 47(D1):D801-D806.

34. Smith CM, Hayamizu TF, Finger JH, Bello SM, McCright IJ, Xu J, Baldarelli RM, Beal JS, Campbell J, Corbani LE et al: The mouse Gene Expression Database (GXD): 2019 update. NUCLEIC ACIDS RES 2019, 47(D1):D774-D779.

35. Krupke DM, Begley DA, Sundberg JP, Richardson JE, Neuhauser SB, Bult CJ: The Mouse Tumor Biology Database: A Comprehensive Resource for Mouse Models of Human Cancer. CANCER RES 2017, 77(21):e67.

36. Williams M: Size and flight capability of Anas chathamica, an extinct duck from Chatham Island, New Zealand. Wildfowl; Wildfowl 65.

37. Stunden $\mathrm{CE}$, Bluhm $\mathrm{CK}$, Cheng KM, Rajamahendran R: Factors affecting reproductive performance in captive mallard ducks. THERIOGENOLOGY 1999, 52(3):435-446.

38. Dial TR, Heers AM, Tobalske BW: Ontogeny of aerodynamics in mallards: comparative performance and developmental implications. The Journal of Experimental Biology 2012, 215(21):3693.

39. Chang W, Xie Q, Zheng A, Zhang S, Chen Z, Wang J, Liu G, Cai H: Effects of aflatoxins on growth performance and skeletal muscle of Cherry Valley meat male ducks. Animal Nutrition 2016, 2(3):186191.

40. Zhang YR, Zhang LS, Wang Z, Liu Y, Li FH, Yuan JM, Xia ZF: Effects of stocking density on growth performance, meat quality and tibia development of Pekin ducks. ANIM SCI J 2018, 89(6):925-930.

41. Weatherbee SD, Behringer RR, Rasweiler JJ, Niswander LA: Interdigital webbing retention in bat wings illustrates genetic changes underlying amniote limb diversification. Proceedings of the National Academy of Sciences 2006, 103(41):15103.

42. Tokita M, Abe T, Suzuki $\mathrm{K}$ : The developmental basis of bat wing muscle. NAT COMMUN2012, 3(1):1302.

43. Ohuchi H, Nakagawa T, Yamamoto A, Araga A, Ohata T, Ishimaru Y, Yoshioka H, Kuwana T, Nohno T, Yamasaki $\mathrm{M}$ et al: The mesenchymal factor, FGF10, initiates and maintains the outgrowth of the chick limb bud through interaction with FGF8, an apical ectodermal factor. DEVELOPMENT 1997, 124(11):2235.

44. Young JJ, Grayson P, Edwards SV, Tabin CJ: Attenuated Fgf Signaling Underlies the Forelimb Heterochrony in the Emu Dromaius novaehollandiae. CURR BIOL 2019, 29(21):3681-3691. 
45. Vishal K, Lovato TL, Bragg C, Chechenova MB, Cripps RM: FGF signaling promotes myoblast proliferation through activation of wingless signaling. DEV BIOL 2020, 464(1):1-10.

46. Peterson SJ, Krasnow MA: Subcellular Trafficking of FGF Controls Tracheal Invasion of Drosophila Flight Muscle. CELL 2015, 160(1):313-323.

47. Pan S, Lin Y, Liu Q, Duan J, Lin Z, Wang Y, Wang X, Lam SM, Zou Z, Shui G et al: Convergent genomic signatures of flight loss in birds suggest a switch of main fuel. NAT COMMUN2019, 10(1):2756.

48. Sackton TB, Grayson P, Cloutier A, Hu Z, Liu JS, Wheeler NE, Gardner PP, Clarke JA, Baker AJ, Clamp $\mathrm{M}$ et al: Convergent regulatory evolution and loss of flight in paleognathous birds. SCIENCE 2019, 364(6435):74.

49. Chen S, Zhou Y, Chen Y, Gu J: fastp: an ultra-fast all-in-one FASTQ preprocessor. BIOINFORMATICS 2018, 34(17):i884-i890.

50. Li H, Durbin R: Fast and accurate short read alignment with Burrows-Wheeler transform. BIOINFORMATICS 2009, 25(14):1754-1760.

51. Li H, Ruan J: Mapping Short DNA Sequencing Reads and Calling Variants Using Mapping Quality Scores. GENOME RES 2008, 18:1851-1858.

52. Danecek P, Auton A, Abecasis G, Albers CA, Banks E, DePristo MA, Handsaker RE, Lunter G, Marth GT, Sherry ST et al: The variant call format and VCFtools. BIOINFORMATICS 2011, 27(15):2156-2158.

53. Coop G, Pickrell JK, Novembre J, Kudaravalli S, Li J, Absher D, Myers RM, Cavalli-Sforza LL, Feldman MW, Pritchard JK: The Role of Geography in Human Adaptation. PLOS GENET 2009, 5(6):e1000500.

54. Purcell S, Neale B, Todd-Brown K, Thomas L, Ferreira MAR, Bender D, Maller J, Sklar P, de Bakker PIW, Daly MJ et al: PLINK: A Tool Set for Whole-Genome Association and Population-Based Linkage Analyses. The American Journal of Human Genetics 2007, 81(3):559-575.

55. Ortiz EM: vcf2phylip v2.0: convert a VCF matrix into several matrix formats for phylogenetic analysis. In., v2.0 edn: Zenodo; 2019.

56. Kozlov AM, Darriba D, Flouri T, Morel B, Stamatakis A: RAxML-NG: a fast, scalable and user-friendly tool for maximum likelihood phylogenetic inference. BIOINFORMATICS 2019, 35(21):4453-4455.

57. Letunic I, Bork P: Interactive Tree Of Life (iTOL) v4: recent updates and new developments. NUCLEIC ACIDS RES 2019, 47(W1):W256-W259.

58. Kim D, Langmead B, Salzberg SL: HISAT: a fast spliced aligner with low memory requirements. NAT METHODS 2015, 12(4):357-360.

59. Liao Y, Smyth GK, Shi W: featureCounts: an efficient general purpose program for assigning sequence reads to genomic features. BIOINFORMATICS 2014, 30(7):923-930.

60. Love MI, Huber W, Anders S: Moderated estimation of fold change and dispersion for RNA-seq data with DESeq2. GENOME BIOL 2014, 15(12):550.

\section{Tables}

Table 1: The candidate SNP wit high fst and pi value, and the nearby genes. 


\begin{tabular}{|c|c|c|c|c|c|}
\hline Chromosome & Position & $\begin{array}{l}\text { Fst } \\
\text { value }\end{array}$ & $\begin{array}{l}\text { Pi value for } \\
\text { wild ducks }\end{array}$ & $\begin{array}{l}\text { Pi value for } \\
\text { domesticated ducks }\end{array}$ & gene \\
\hline NC_040047.1 & 117517559 & 1.00 & 0.00 & 0.00 & FAM110B \\
\hline NC_040059.1 & 13649779 & 0.98 & 0.03 & 0.00 & LOC101797191 \\
\hline NC_040059.1 & 13664091 & 0.95 & 0.08 & 0.00 & \\
\hline NC_040046.1 & 140162105 & 0.90 & 0.09 & 0.08 & WASHC1 \\
\hline NC_040059.1 & 14882712 & 1.00 & 0.00 & 0.00 & ETF1 \\
\hline NC_040047.1 & 151884726 & 1.00 & 0.00 & 0.00 & TRAPPC9 \\
\hline NC_040054.1 & 17983955 & 1.00 & 0.00 & 0.00 & OTOS \\
\hline NC_040053.1 & 18334829 & 0.96 & 0.06 & 0.00 & \\
\hline NC_040059.1 & 18701490 & 1.00 & 0.00 & 0.00 & \\
\hline NC_040059.1 & 18703887 & 0.95 & 0.06 & 0.00 & \\
\hline NC_040048.1 & 24832756 & 1.00 & 0.00 & 0.00 & LOC106018338 \\
\hline NC_040046.1 & 26670458 & 1.00 & 0.00 & 0.00 & TMEM272 \\
\hline NC_040047.1 & 32308688 & 0.96 & 0.06 & 0.00 & ABCB5 \\
\hline NC_040069.1 & 3371512 & 1.00 & 0.00 & 0.00 & \\
\hline NC_040048.1 & 38299125 & 0.96 & 0.06 & 0.00 & THADA \\
\hline NC_040059.1 & 4696939 & 1.00 & 0.00 & 0.00 & UBE2B \\
\hline NC_040046.1 & 46978636 & 0.95 & 0.04 & 0.07 & \\
\hline NC_040046.1 & 55294716 & 0.94 & 0.07 & 0.00 & FGF14 \\
\hline NC_040046.1 & 55299005 & 0.90 & 0.10 & 0.08 & FGF14 \\
\hline NC_040072.1 & 5755676 & 1.00 & 0.00 & 0.00 & UBE2T \\
\hline NC_040059.1 & 735649 & 0.93 & 0.05 & 0.09 & LOC113845140 \\
\hline NC_040047.1 & 95954650 & 1.00 & 0.00 & 0.00 & LOC113842815 \\
\hline
\end{tabular}

\section{Figures}


A

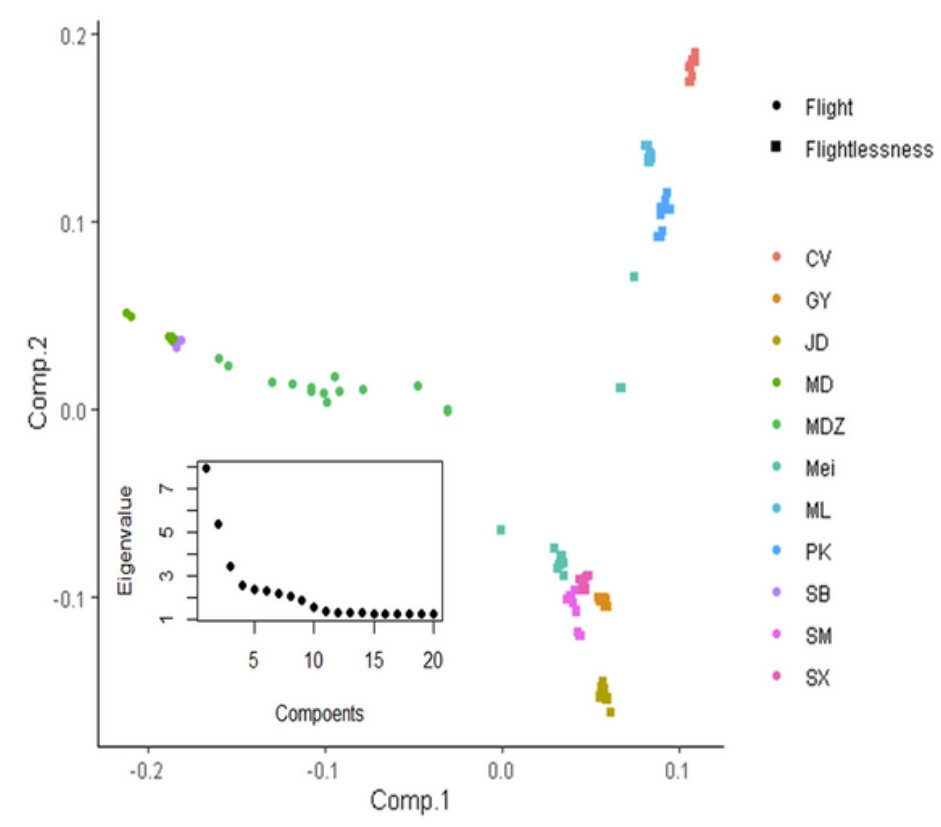

B
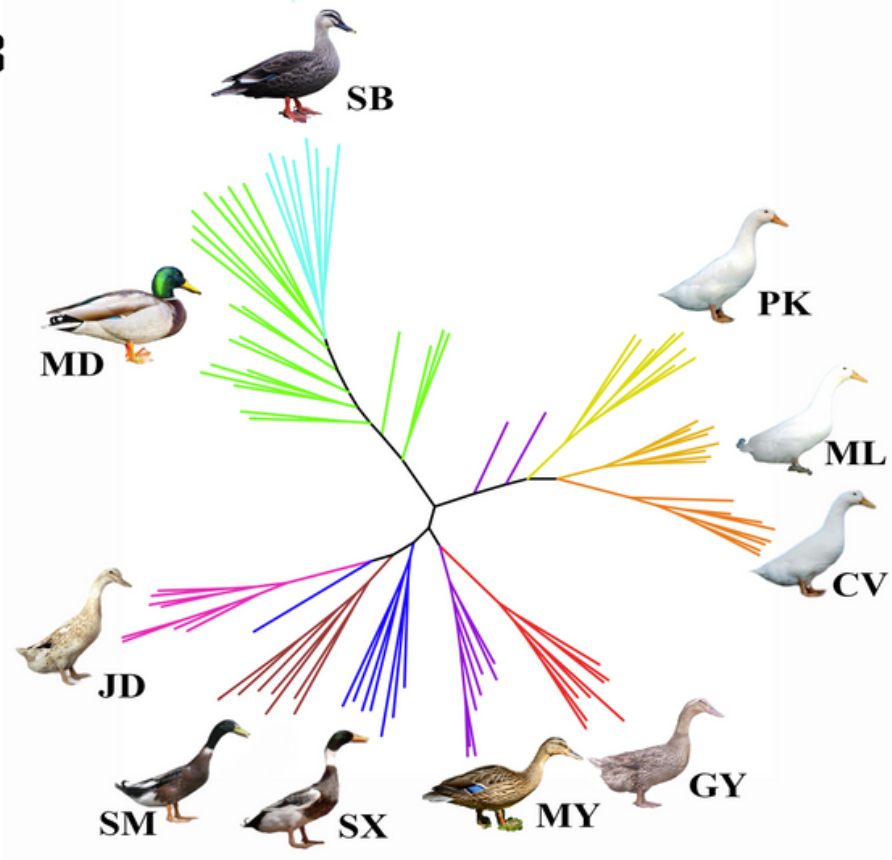

Figure 1

Population structure of ducks. (A) The PCA plot of ducks, the inner dot plot represents eigenvalue of top 20 principal component. (B) The maximum likelihood phylogenetic tree of all ducks.

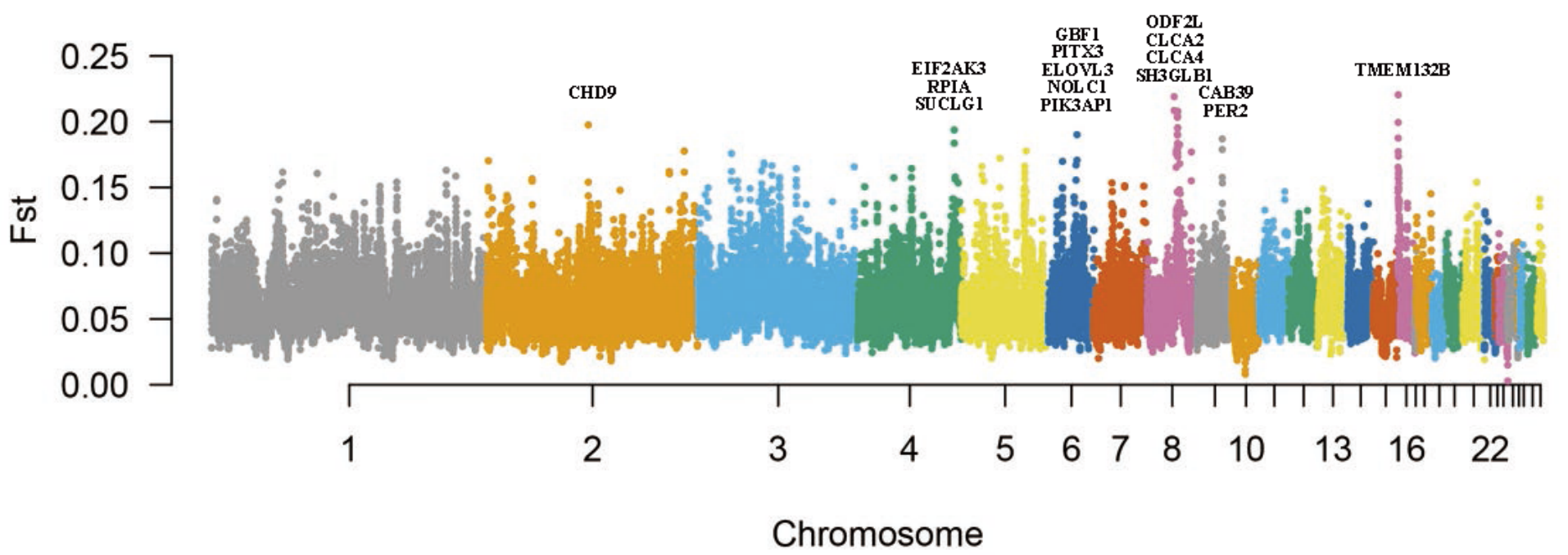

Figure 2

The Manhattan plot and genes that overlapping with sweep peaks. 


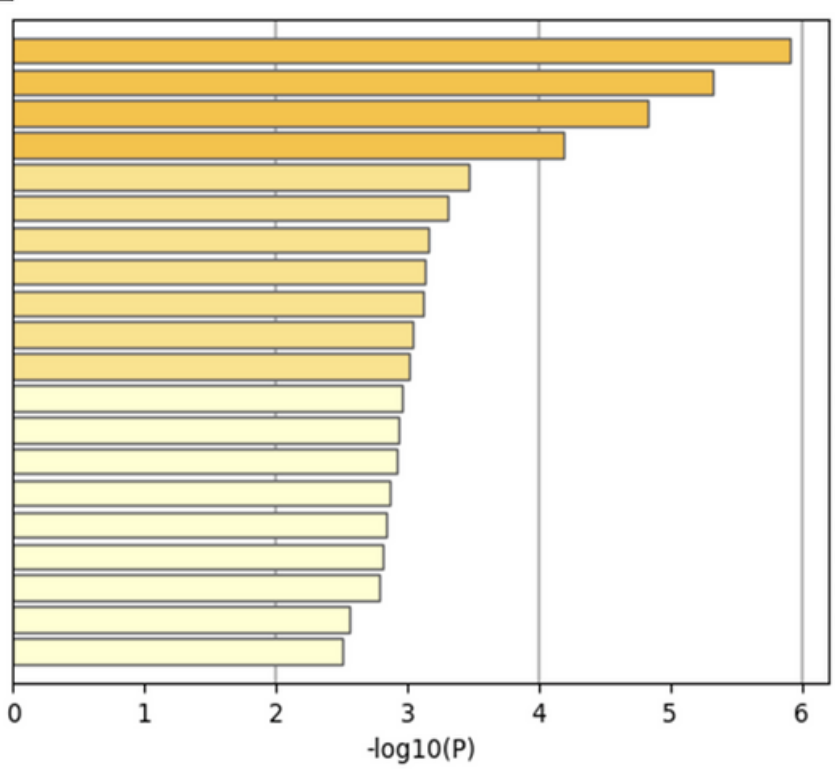

B

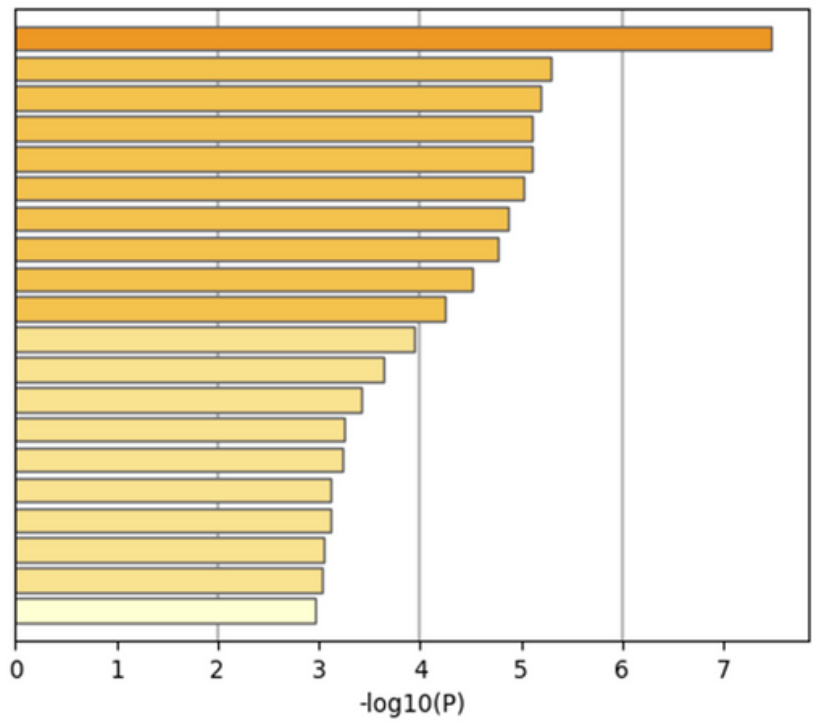

R-HSA-1500931: Cell-Cell communication CORUM:2850: ITGA5-ITGB1-FN-1-NOV complex R-HSA-8874081: MET activates PTK2 signaling GO:0036507: protein demannosylation R-HSA-196849: Metabolism of water-soluble vitamins and cofactors GO:0042559: pteridine-containing compound biosynthetic process GO:0071347: cellular response to interleukin-1 GO:0098742: cell-cell adhesion via plasma-membrane adhesion molecules GO:0030029: actin filament-based process

R-HSA-8953854: Metabolism of RNA

GO:0006403: RNA localization

GO:0090278: negative regulation of peptide hormone secretion GO:0045056: transcytosis

R-HSA-194315: Signaling by Rho GTPases

GO:0099515: actin filament-based transport GO:0006855: drug transmembrane transport GO:0032402: melanosome transport GO:0001501: skeletal system development GO:0006631: fatty acid metabolic process R-HSA-9660821: ADORA2B mediated anti-inflammatory cytokines production
GO:0001503: ossification GO:1905517: macrophage migration GO:0009074: aromatic amino acid family catabolic process GO:0010720: positive regulation of cell development GO:1903522: regulation of blood circulation GO:0035973: aggrephagy GO:0043269: regulation of ion transport GO:0001501: skeletal system development GO:0006935: chemotaxis GO:0007507: heart development GO:0007169: transmembrane receptor protein tyrosine kinase signaling pathway hsa04080: Neuroactive ligand-receptor interaction GO:0051051: negative regulation of transport GO:0006970: response to osmotic stress GO:0051250: negative regulation of lymphocyte activation GO:0048608: reproductive structure development GO:0001764: neuron migration GO:0040008: regulation of growth GO:0043062: extracellular structure organization GO:0001823: mesonephros development

\section{Figure 3}

Gene enrichment analysis for DEGs. (A) GO analysis for slide windows algorithm. (B) GO analysis for DEGs in breast muscle. 

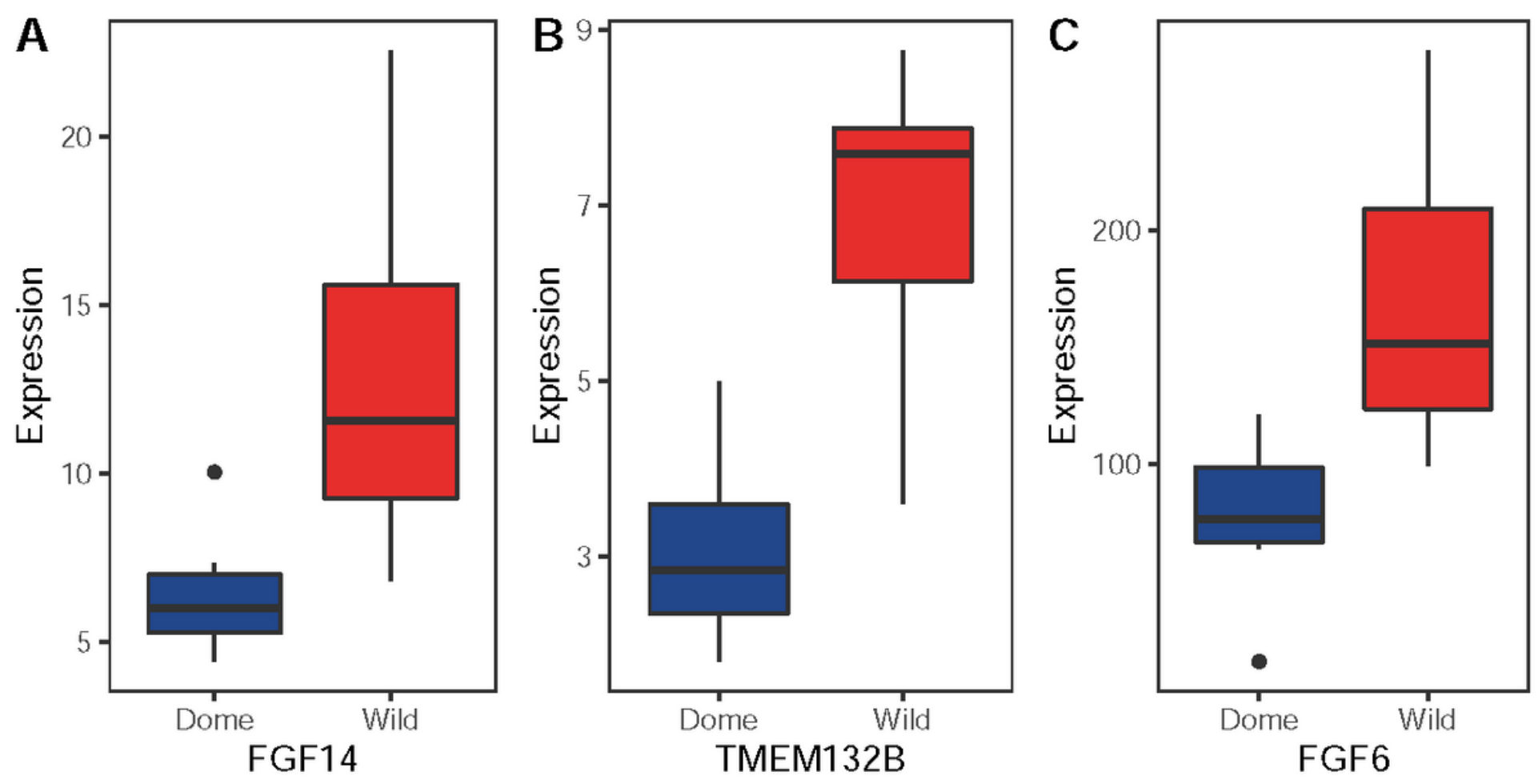

Figure 4

Expression levels of FGF14, FGF6 and TMEM132B in breast muscle between the wild (Wild) and domesticated ducks (Dome). 